\title{
FACILITATING INTEGRATED SERVICE DELIVERY VIA NETWORKING FORUMS: LESSONS FROM A CASE STUDY
}

\section{Victor Chikadzi, Sarah Mafetsa}

\section{INTRODUCTION}

At the dawn of democracy in South Africa in 1994 the government inherited a welfare system that was fragmented because of apartheid institutional arrangements. Collaboration between different government departments and organisations within civil society was almost non-existent. A silo culture was prevalent as a result of haphazard service delivery efforts, which led to ineffective and inefficient service delivery. Given the disjointed nature of service delivery within social welfare as well as other sectors of the economy, urgent measures were needed to ensure that the population benefits from well-coordinated and holistic service delivery efforts (White Paper for Social Welfare, 1997). It is in this context that the notion of integrated service delivery (ISD) became a national buzzword. There was political pressure for government departments as well as organisations within civil society to ensure that integration of services was promoted at all costs. As a part response to this call, the Department of Social Development (DSD) introduced the idea of networking forums. Several government departments and non-governmental organisations (NGOs) participate in these networking forums. Monthly meetings are held where stakeholders from different NGOs and government departments discuss and formulate coordinated responses to service delivery within particular geographical areas of operation. Thus, through these monthly meetings a platform for networking between NGOs, the DSD and other government departments is created which allows sharing of information and initiation of coordinated efforts in serving the communities within their demarcated radius of operation. This article examines how these networking forums pioneered by the DSD have been instrumental in enhancing integrated service delivery.

\section{INTEGRATED SERVICE DELIVERY (ISD)}

ISD is used to refer to the collaborative rendering of social services among institutions which results in benefits for the service users as well as the service providers (Kernaghan, 2005; Munday, 2007). According to Kodner and Spreeuwenberg (2002:3), ISD is "a coherent set of methods and models on the funding, administrative, organisational, and service delivery and clinical levels designed to create connectivity, alignment and collaboration within and between the cure and care sectors". Similarly, Contandriopoulos, Denis, Touati and Rodriguez (2003:8) observe that "integration is the process that involves creating and maintaining, over time, a common structure between independent stakeholders (and organisations) for the purpose of coordinating their interdependence in order to enable them to work together on a collective project". IDS is rooted within the systems thinking perspective, which is a way of looking at phenomena in their holistic context. Within systems thinking there is an understanding that every organisation, while being a complete unit unto itself, is always a part of a bigger system within which there are other organisations. These organisations are not independent of each other in that what happens within an organisation in a way affects or is affected by 
what happens in other organisations. The idea behind ISD is that organisations that render social services are sub-systems of a bigger system of social service delivery within a particular geographical area. If there is to be smooth functioning within the bigger system, the sub-systems ought to interact in a harmonious way while maintaining their identity (wholeness) as sub-systems (Kodner \& Spreeuwenberg, 2002). Contrary to the idea of rivalry that prevails amongst private sector organisations, ISD seeks to foster cooperation and mutual dependence amongst social service agencies in a manner that results in benefits for clients and the agencies involved (Contandriopoulos et al., 2003).

There are two common ways through which integration of services occurs, which could be horizontal or vertical integration. Vertical integration is prevalent in health care settings and it refers to the amalgamation of the different hierarchical levels of services within a social service system. An example of this could be an amalgamation of hospital, clinic and community-based health services into one unit to ensure a continuum of care under one roof (England \& Lester, 2005). There is also horizontal integration. Munday (2007) observes that horizontal integration is the joining up of services, organisations and professions within and across different sectors of the economy that were previously working in isolation. The need for horizontal integration arises in situations where organisations have to respond to the plight of service users who are exposed to a multiplicity of problems. This is done to achieve economies of scale that come with better coordination and resource allocation when organisations work together (Munday, 2007).

There are also various forms through which integration of services occurs, other than horizontally and vertically. According to Richardson and Patana (2012), collocation, collaboration and cooperation or combinations of these are the main forms through which integration occurs. These forms of integration are explained below.

\section{Collocation}

"Collocation refers to having all agencies in one location such as: legal services, mental health services, health services, housing services, social services or case management services" (Richardson \& Patana, 2012:4). The main advantage of collocation is that when services providers are clustered in one location, service users can reduce the time and travel costs, given that they would not have to travel to different locations to access interrelated services. For example, a person suffering from a mental health-related problem will most likely make use of heath care services as well, and so having such services clustered saves the person time and money. On the other hand, collocation may lead to increased effectiveness and efficiency amongst the agencies involved as they are more likely to collaborate given that the distance barrier will be out of the way (Sloper, 2004).

\section{Collaboration}

According to Sloper (2004), collaboration is more advanced form of integration than collocation. "It refers to agencies working together through information sharing and training, and creating a network of agencies to improve service user experience. Collaboration is a necessary step for reducing the gaps in services for service users. By sharing knowledge, agencies and professionals can improve the referral process to other services offered" by different agencies (Richardson \& Patana, 2012:4). 


\section{Cooperation}

Rosenheck et al. (2003 as cited in Richardson \& Patana, 2012) note that cooperation is the highest degree of integration. Cooperation is denoted in instances where professionals from different settings and backgrounds communicate and work together in a team effort to tackle or address clients' problems. According to Richardson and Patana, (2012) in this form of integration communication is regarded as a vital ingredient. They further note that effective cooperation occurs because of good communication, and this is key to enhancing service users' outcome. "When professionals work well together, costs can be lowered, services are not duplicated, and the identification and response to service user needs can occur more quickly" (Richardson \& Patana, 2012:4).

\section{THE IMPORTANCE OF INTEGRATED SERVICE DELIVERY}

\section{Reduction of costs}

Richardson and Patana (2012) argue that one of the major reasons that have been put forward in favour of ISD is the issue of cost effectiveness. When services are integrated service users can save on a lot of unnecessary expenditure by accessing a diverse range of services in one location. In clinical settings, "through an integrated approach, service users can save money by accessing multiple services in one place, or by reducing other transaction costs (telephone calls, other communications, time and working hours). Moreover, the overall expenditure on services can also be brought down by reducing hospitalisation and duplication of services" (Richardson \& Patana, 2012:12). Similarly, integration of services helps to curtail over-use of services because of the effective coordination that occurs in an integrated continuum of care (Grone \& Garcia-Barbero, 2001). On the other hand, (Richardson \& Patana, 2012:15) note that "the integration of services, through effective collaboration in particular, will have the dual benefits of reducing gaps in priority services, and avoiding duplication of generic services from different agencies".

\section{Easy accessibility}

The question of accessibility has also been put forward as a key justification for promoting ISD. According to Richardson and Patana (2012:15), "accessibility refers to the ease, and the extent, of access service users have to the services for which they are eligible". In most cases users of social services are usually those that suffer from a range of disadvantages and vulnerabilities. These people tend to have a limited understanding of the care system itself, which more often than not is complex. This limited understanding may lead to their failing to navigate the system and they fail to access the full range of benefits available to them. This results in reduced quality of care for vulnerable populations. To this end, integrated services are critical in order to overcome the barriers that vulnerable populations may face when having to navigate disjointed and complex systems of care.

\section{Improved quality of services}

Several studies (OECD, 2012; Verstappen, 2003) have shown that ISD leads to a higher quality of services than when organisations offer services in a disjointed manner. When 
people from different professional backgrounds such as doctors, social workers and psychologists work together, it leads to improved outcomes for the service user, while improving the understanding that each professional will have of the complexity of social pathologies that confront service users. This sharpens attention to detail, which allows for a holistic response to helping service users. Such outcomes may not be possible when professionals work in isolation. For example, a doctor working in isolation to treat a patient suffering from depression may seek to administer drugs as the only remedy. While depression is considered a disease, its trigger may be a personal problem which the service user needs to be helped to solve. A holistic approach to integrated services will enable the service user to get the help of a social worker and a psychologist to tackle the cause of the problem, given that depression is only a symptom of an underlying cause which may be a personal problem that a doctor would not ordinarily seek to tackle. It would therefore be logical that the doctor would treat the patient and then make a referral for the patient to seek further help from other professionals. In this case, ISD allows the service user to access holistic and good-quality intervention (Verstappen, 2003). Similarly, research done by OECD (2012) showed that children who experienced mental health problems benefited more when mental health services where integrated within education institutions. On the other hand, better-quality services are delivered in cases where a professional speaks on behalf of a service user than when service users advocate for themselves (Richardson \& Patana, 2012).

\section{RESEARCH QUESTION}

- What are the stakeholders' perceptions of how the Nelmapius networking forum enables integrated service delivery and partnerships among participant organisations?

\section{RESEARCH METHODOLOGY}

\section{Aim of the study}

The aim of the study was to explore the perceptions of the stakeholders on the role and effectiveness of networking forums in promoting integrated service delivery within and between government departments and NGOs engaged in delivering welfare services.

\section{Research design}

A qualitative research approach was adopted. The use of a qualitative research approach enables researchers to obtain a rich and in-depth understanding of the phenomenon being studied. Within the qualitative research paradigm adopted by the study, a case study research design was utilised. According to Yin (1994:13), a case study is "an empirical inquiry that investigates a contemporary phenomenon within its life context, especially when the boundaries between phenomenon and context are not clearly evident". For the purposes of this study the Nelmapius networking forum was chosen for study in order for the researchers to understand the extent to which networking forums facilitated integrated service delivery. 


\section{Sample and sampling procedure}

A sample of 15 participants was selected for the study using purposive sampling, which is a method that is reliant upon the researcher's mature judgment in choosing participants from whom the most valuable data that address the research objectives can be yielded (Merriam, 1998). Ten of the participants were social workers who participated in the Nelmapius forum. The other five participants were senior managers, of whom two were from the Department of Social Development and the other three were selected from the NGOs that took part in the forum.

\section{Data-collection method and tools}

In-depth interviews were used in gathering data for the research. The advantage of using interviews is that they allow detailed data to be collected, while the flexibility of interviews allows the researcher to probe for more detail as well as asking for clarification during the interview (Greeff, 2002). A semi-structured interview schedule is also helpful as a tool to guide and focus the interview and ensure that the researcher does not omit to ask questions that are critical in addressing the research objectives (Patton, 2002). Each interview took approximately 45 minutes to an hour to complete and data were collected over a period of one month. The interviews were spaced according to the availability of participants. While a focus group discussion would have been ideal for this study, the method wasn't utilized because of the participants' concerns about confidentiality.

\section{Data analysis}

Thematic content analysis was used during data analysis. Patton (2002) observes that thematic content analysis refers to analysing textual data gathered during field work. It is a qualitative data-reduction and sense-making effort that takes a volume of qualitative material and attempts to identify core consistencies and meanings, which become the themes that guide the write-up process (Patton, 2002).

\section{RESULTS AND DISCUSSION}

\section{Networking forums are a foundational basis that leads to active engagement amongst various organisations and stakeholders within the social service sector.}

The findings of the study clearly show that the networking forum which was initiated by the Department of Social Development was a key development in kick-starting integrated service delivery amongst organisations within the Nelmapius region. Participants noted that before the initiation of the networking forum, virtually all organisations worked apart from each other. There was little meaningful contact amongst NGOs working in the Nelmapius region. Participants noted that isolation and rivalry among organisations was a characteristic feature. The only contact that was reported was communication between NGOs and the DSD, which only happened because the DSD funds the NGOs operating in the Nelmapius area. Thus contact was limited to a low-key engagement level, which took place when NGOs received funds and when reporting on the use of funds to the DSD. This is clearly evidenced in the participants' accounts; one participant asserted: 
"In the past we used to communicate with the department during the period of submitting business plans and our reports for funding review. We mainly met with the Department when we needed some clarity on funding related issues, but there was no platform whereby we would sit down as NGOs and the Department to discuss issues in the community."

Similarly another participant mentioned that:

"The relationship that we had with the Department was not enough, because we only contacted them to inquire about funding issues, but there was no space to discuss other issues. We also did not know who to contact when we wanted to inquire about certain issues. The forum is a meeting point between government and NGOs, because that is where we would sit as stakeholders in the Nelmapius community and discuss community needs and the ways to address them."

It is apparent from the participants' accounts above that before the commencement of the Nelmapius networking forum, a silo culture was prevalent among organisations working in the Nelmapius region. It is also clear that after the Nelmapius networking forum was initiated, there was a platform through which organisations began to converse with each other. Gradually relationships began to form as common ground emerged on key service delivery issues that these organisations could work together on. It is our contention that, if integrated service delivery is to be realised, there is a need to put deliberate effort into promoting it. Put simply, integrated service delivery is not an automatic process; it needs intentionality. We therefore argue that the networking forum is an effective mechanism to facilitate the delivering of integrated services by the organisations.

\section{Collaboration}

The initial manifestation of integrated service delivery amongst social service agencies that worked within the Nelmapius community was evident at the level of collaboration, which according to Sloper (2004) is an advanced form of integration. Collaboration is evidenced when organisations share information and other resources as they work together to enhance service users' experiences. The collaboration experience that developed among stakeholders in the Nelmapius networking forum is evident from the accounts given by participants. One participant argued that:

"The forum helped us to be alert of the issues in the community because if one organisation has noticed some trend in the community, they would discuss it in the forum and all the forum members would engage effectively on the issue."

Similarly, another participant mentioned that:

"There was a time when one organisation did not have a social worker and as a result they were experiencing difficulties in terms of providing services to the community. Other organisations were able to assist that organisation by providing their social workers until such time that the organisation was able to employ a social worker." 
It is evident from these participants' accounts that the networking forum enabled organisations to begin sharing information and discussing key service delivery issues. Participants spoke of a silo culture as having existed prior to the establishment of the forum. Once the forum was established, tangible results were also noticeable as evidenced by the sharing of information, among other things. This would ordinarily not have happened, had it not been for the networking forum.

\section{Partnership building}

One of the notable features that came about as a result of the Nelmapius networking forum was the ability to foster partnerships amongst participant organisations when delivering services to the community. Participants mentioned that the Nelmapius forum provided an opportunity for various stakeholders from NGOs and the DSD to partner and work together when rendering services in the Nelmapius community. Through the networking forum participating stakeholders were able to identify areas of service delivery which they could collectively plan and jointly implement. This is clearly evidenced in the view by one participant who asserted that:

"As forum members we were able to plan jointly as stakeholders in the forum in terms of organising community awareness campaigns. We worked together in terms of planning, organising and implementing the campaigns."

In the same vein one participant mentioned that:

"I remember the time we were celebrating the mental health awareness month in our organisation. We invited the members of the forum and they helped us a lot in terms of manpower. When we organised community awareness events all the organisations were given different tasks to ensure that each organisation participates and during the day of the event each organisation will have their own stall to advertise their services and they will also bring pamphlets and leaflets for community members."

The participants' accounts above clearly show that the establishment of the networking forum was instrumental in ending the isolation and rivalry that tended to characterise relationships amongst stakeholders in the period before the networking forum was established. The partnerships that organisations entered into, as they worked together, are a clear manifestation of cooperation, which is the highest degree of integration that can be achieved in integrated service delivery (Rosenheck et al., 2003, as cited in Richardson \& Patana, 2012). Without the networking forum, it is difficult to imagine that such levels of cooperation could ever have been achieved within the social service agents operating in the Nelmapius region. It is therefore beyond doubt that networking forums are a mechanism through which integrated service delivery can be facilitated.

\section{The evidence that organisations and clients benefited from integrated services delivery}

After the establishment of the Nelmapius networking forum, organisations were able to offer integrated services to clients within the Nelmapius region. This process benefited 
the organisations involved as well as their clientele in several ways. These are discussed below.

\section{Curbing duplication of services}

Participants stated that when the Nelmapius forum was initiated, one of the critical issues which had to be dealt with urgently was the prevention of duplication of services. They pointed out that when they first met as forum members, they did not know each other and also they did not know the specific areas of operation of each organisation in the Nelmapius area. After the establishment of the forum the participants realised the need to divide and allocate geographical areas of operation. They stated that it is within the forum that they were able to clarify the services of each organisation and negotiate the areas of operation. One participant stated that:

"In the beginning we were just working haphazardly, we did not know each other as service providers in the Nelmapius area and also did not know which organisations were providing services to other sections of Nelmapius. When we met as forum members we divided the sections accordingly between the organisations. We also drafted a working agreement between all organisations and as a result we were able to know which organisation is responsible for which section."

Another participant stated that:

"Dividing the sections and knowing our areas of jurisdiction helped to improve service delivery because we were able to control our services and prevent our clients from misusing the system because sometimes a client will go to one organisation to request a food parcel and do the same with another organisation."

It is apparent from these selected accounts from participants that integrated service delivery which came to fruition as a result of the establishment of the forum benefited organisations in many ways. Firstly, organisations were saved a lot of time by having to focus on a smaller radius of operation than when they had previously delivered services to the whole Nelmapius community. No doubt this helps organisations to be more focused and they get to know their clientele base better. This in turn leads to improved services. On the other hand, a lot of wasteful expenditure was curbed when organisations demarcated focus areas among themselves. This helped to avoid "double dipping" by clients, who used to claim the same service from two or more organisations. The networking forum enabled organisations to bring better coordination of service delivery efforts and to curtail rampant abuse of social services delivery systems that were in disarray. This finding corroborates the view by Grone and Garcia-Barbero (2001), who note that integration of services helps to curtail over-use of services as a result of the effective coordination that is possible in an integrated continuum of care.

\section{Improved effectiveness in service rendering}

Apart from curbing duplication of services by organisations, the establishment of the Nelmapius networking forum helped organisations to improve on the quality of services 
they rendered to participants. Two significant developments were instrumental in this regard. Firstly, some organisations agreed to move from generic services to offering specialised services. Secondly, information sharing led to increased benefits for clients through an informed referral system. This is apparent in the selected accounts given by participants below. One participant stated that:

"As participants of the Nelmapius forum we were able to co-operate and work effectively in order to improve our services in the community. Through the forum one of the organisations agreed to handle all substance abuse cases in the whole of Nelmapius area irrespective of the jurisdiction, as a result all substance abuse related cases were handled by them. Another organisation also agreed to handle all the adoptions cases in the Nelmapius area."

The offering of specialised services by some of the organisations that participated in the forum as evidenced in the account above led to dual benefits. The first benefit derived from offering specialised services was that personnel working in these organisations became very good at and more knowledgeable about the services. This in turn translated into improved services for clients. On the other hand, it reduced the work load in other organisations, which gave them time to focus on other key service delivery areas.

Another participant stated that:

"The forum helped us to know each other and the area of operation for each service organisation. The working agreement was a very fruitful tool because I was sure of where, when and to whom I should refer the clients. This helped me a lot because clients were not sent from pillar to post."

It is also clear from this participant's account that information sharing led to improved knowledge and awareness amongst service providers about the services of other organisations in the area. This led to accurate and timely referrals for clients. This no doubt improves the service users' experiences much more than when service providers are ignorant about what other organisations are doing.

\section{Capacity building}

Capacity building is also one of the key benefits that were derived by participants of the Nelmapius forum. As organisations worked together, participants consisted of auxiliary social workers, social workers, senior social workers and social work supervisors. Through forum meetings as well as other contexts when members worked collectively, members were able learn from the experience and expertise of others. This helps to improve the capacity of personnel compared to when they work in isolation from fellow professionals. One participant noted that:

"We were learning a lot in the forum because the Department of Social Development would normally conduct training about Acts and Legislation to keep us updated. This really helped us to be aware of the Department's expectations and had a positive impact in our rendering of service to the community." 
It is clear from this participant's account above that integrated service delivery allows for "cross-pollination" of views, experiences and skills which leads to capacity building. According to Glickman and Servon (2010), when people interact and work together, such a process facilitates the exchange of skills, experiences and resources, which in turn leads to increased competence and efficiency amongst members. Without doubt this is clearly evidenced in the work of the forum.

\section{CONCLUSIONS}

The discussion above has examined how the Nelmapius networking forum led to the realisation of integrated service delivery amongst various organisations working in the Nelmapius region. The period before the establishment of the forum was characterised by isolation and rivalry amongst organisations, while the networking forum managed to bring these organisation together. This process led to collective service delivery amongst various stakeholders. The process led to various benefits such as the curbing of duplication services, improved coordination and delivery of services, cost effectiveness and capacity building amongst organisations that participated. This case study demonstrates that networking forums can be an effective platform from which to kickstart service integration amongst organisations within and between different sectors. Integrated service delivery does not happen in and of itself. It has to be deliberately facilitated. Given the current silo culture that characterises government departments and NGOs that work directly and indirectly within the welfare sector, we argue that networking forums can form the bedrock upon with to facilitate cross-sectoral partnerships and collaborations. This can go a long way towards plugging service delivery gaps that are characteristic of the South African welfare context. Fragmentation of welfare service delivery must be overcome if communities are to benefit from holistic and better quality services from government, the private sector and NGOs. To this end, networking forums can be instrumental in fostering integrated service delivery.

\section{REFERENCES}

DEPARTMENT OF WELFARE AND POPULATION DEVELOPMENT. 1997. White Paper for Social Welfare: General notice 1108 of 1997. Pretoria: Government printers.

CONTANDRIOPOULOS, A., DENIS, J., TOUATI, N. \& RODRIGUEZ, C. 2003. The integration of health care. Working paper. [Online] Available: http://www.getoss.enap.ca/ GETOSS/Publications/Lists/Publications/Attachments/152/The\%20integration\%20of\%20h ealth\%20care\%20Dimensions\%20and\%20implementation.pdf. [Accessed: 12/02/2013].

ENGLAND, E. \& LESTER, H. 2005. Integrated Mental Health Services in England: a policy paradox. International Journal of Integrated Care, 5. [Online] Available: http://www.ncbi.nlm.nih.gov/pmc/articles/PMC1475728/pdf/ijic2005-200524.pdf.

[Accessed: 12/02/2013].

GLICKMAN, N.J. \& SERVON, L.J. 2010. More than bricks and sticks: five components of community development corporation capacity. [Online] Available: http://www.tandfonline.com/doi/abs/10.1080/10511482.1998.9521306.

[Accessed: 03/02/2013]. 
GREEFF, M. 2002. Information collection interviewing. In: DE VOS, A.S, STRYDOM, H., FOUCHÉ, C.B. \& DELPORT, C.S.L. (eds) Research at grass roots: for the social sciences and human service professions. Pretoria: Van Schaik Publishers.

GRONE, O. \& GARCIA-BARBERO, M. 2001. Integrated care: a position paper of the WHO European office for integrated health care services. International Journal of Integrated Care, 1. [Online] Available: http://www.ijic.org/index.php/ijic/article/ view/28/56. [Accessed: 23/02/2013].

KODNER, D.L. \& SPREEUWENBERG, C. 2002. Integrated care: meaning, logic, applications, and implications - a discussion paper. International Journal of Integrated Care, 2. [Online] Available: http://www.ncbi.nlm.nih. gov/pmc/articles/PMC1480401/. [Accessed: 23/02/2013].

MERRIAM, S.B. 1998. Case study research in education: a qualitative approach. San Francisco: Jossey- Bass.

MUNDAY, B. 2007. Integrated social services in Europe. Council of Europe Publishing.

OECD. 2012. Sick on the Job? Myths and realities about mental health and work. [Online] Available: http://www.weekmentalegezondheid.nl/doc/sick_on the_Job.pdf. [Accessed: 03/02/2013].

PATTON, Q.R. 2002. Qualitative research and evaluation and methods. California: Sage Publications.

RICHARDSON, D. \& PATANA, P. 2012. Integrating service delivery: why, for who and when. [Online] Available: http://www.oecd.org/els/soc/richardson_patana\%20 integrating $\% 20$ service $\% 20$ delivery $\% 20$ why $\% 20$ for $\% 20$ who\%20and\%20how.pdf. [Accessed: 03/02/2013].

SLOPER, P. 2004. Facilitators and barriers for co-ordinated multi-agency services. Child Care, Health and Development, 30(6):565-740. [Online] Available: http://onlinelibrary. wiley.com/doi/10.1111/j.1365-2214.2004.00468.x/pdf. [Accessed: 03/02/2013].

VERSTAPPEN, S.M.M. 2003. Effectiveness and costs of multidisciplinary care in patients with rheumatoid arthritis. International Journal of Integrated Care, 3. [Online] Available: Retrieved from http://www.ijic.org/index.php/ijic/article/view/83/165.

[Accessed: 12/02/2013].

YIN, R.K. 1994. Case study research: design and methods. Thousand Oaks: Sage Publications.

Dr Victor Chikadzi, School of Human and Community Development, Department of Social Work, University of the Witwatersrand; Ms Sarah Mafetsa, Postgraduate Student, Department of Social Work, University of the Witwatersrand, Johannesburg, South Africa. 